\title{
REVIEW
}

\section{Strategies for combating bacterial biofilm infections}

\author{
Hong Wu ${ }^{1,2}$, Claus Moser ${ }^{1}$, Heng-Zhuang Wang ${ }^{1}$, Niels Høiby ${ }^{1,2}$ and Zhi-Jun Song ${ }^{1,3}$
}

Formation of biofilm is a survival strategy for bacteria and fungi to adapt to their living environment, especially in the hostile environment. Under the protection of biofilm, microbial cells in biofilm become tolerant and resistant to antibiotics and the immune responses, which increases the difficulties for the clinical treatment of biofilm infections. Clinical and laboratory investigations demonstrated a perspicuous correlation between biofilm infection and medical foreign bodies or indwelling devices. Clinical observations and experimental studies indicated clearly that antibiotic treatment alone is in most cases insufficient to eradicate biofilm infections. Therefore, to effectively treat biofilm infections with currently available antibiotics and evaluate the outcomes become important and urgent for clinicians. The review summarizes the latest progress in treatment of clinical biofilm infections and scientific investigations, discusses the diagnosis and treatment of different biofilm infections and introduces the promising laboratory progress, which may contribute to prevention or cure of biofilm infections. We conclude that, an efficient treatment of biofilm infections needs a well-established multidisciplinary collaboration, which includes removal of the infected foreign bodies, selection of biofilm-active, sensitive and well-penetrating antibiotics, systemic or topical antibiotic administration in high dosage and combinations, and administration of anti-quorum sensing or biofilm dispersal agents. International Journal of Oral Science (2014) 7, 1-7; doi:10.1038/ijos.2014.65; published 12 December 2014

Keywords: antibiotic resistance; antimicrobial treatments; bacterial biofilm; chronic infection

\section{INTRODUCTION}

A structured consortium attached on a living or inert surface formed by microbial cells sticked to each other and surrounded by the selfproduced extracellular polymeric matrix is known as biofilm. The formation of biofilm is considered an adaptation of microbes to hostile environments. ${ }^{1-2}$ A typical development of biofilm-taking Pseudomonas aeruginosa as an example_includes several stages, i.e., attachment to a surface; formation of microcolonies; development of young biofilm; differentiation of structured mature biofilm, and dispersal of mature biofilm. ${ }^{2-4}$ Experimental evidences of $P$. aeruginosa in vitro and in vivo demonstrated clearly that biofilm bacterial cells are significantly more resistant to antibiotics and host immune defense than their planktonic counterparts. ${ }^{4-7}$ Aggressive and intensive antibiotic treatment is usually helpful to control the exacerbations of chronic biofilm infections induced by dispersed bacteria and reduce the biofilms, but can not eradicate the biofilm infections, ${ }^{7-8}$ because the minimal concentration of antibiotic for eradication of mature biofilm is difficult to reach in vivo. ${ }^{5}$ Therefore, once a bacterial biofilm infection established, it becomes difficult to eradicate. Bacterial biofilm formation is widely found in natural environments with water, and also in human diseases, especially in the patients with indwelling devices for the purpose of medical treatments. ${ }^{2,7}$ With the progress of medical sciences, more and more medical devices and/or artificial organs are applied in the treatment of human diseases. However, as a consequent, bacterial biofilm infections become also frequent. It has been reported that vast majority, if not all, of the medical devices or prostheses may result in biofilm infections, which include intravenous catheters, ${ }^{9}$ vascular prosthesis, ${ }^{10}$ cerebrospinal fluid shunts, ${ }^{11}$ prosthetic heart valves, ${ }^{12}$ urinary catheters, ${ }^{12}$ joint prostheses and orthopedic fixation devices, ${ }^{13}$ cardiac pacemakers, ${ }^{14}$ peritoneal dialysis catheters, ${ }^{15}$ intrauterine devices,${ }^{16}$ biliary tract stents, ${ }^{17}$ dentures, ${ }^{18}$ breast implants, ${ }^{19}$ contact lenses ${ }^{20}$ and in the dental area caries and periodontitis, and so on. In addition, there are also biofilm infections not associated with foreign bodies, such as chronic airway infections in cystic fibrosis (CF) patients ${ }^{21}$ or patients with chronic obstructive pulmonary diseases, ${ }^{22}$ native valve endocarditis, chronic otitis media, ${ }^{23}$ chronic sinusitis ${ }^{24}$ and chronic (diabetes) wound infections. ${ }^{25-26}$ It has been estimated that most bacterial infections in human are correlated with biofilm and about $50 \%$ of the nosocomial infections are indwelling devices-associated. ${ }^{27}$

Bacterial biofilms are characterized as highly resistant to antibiotic treatment and immune responses. ${ }^{7}$ Although it is well known that antibiotic treatment is currently most important and effective measure for the control of microbial infections, however, antibiotic treatments are almost impossible to eradicate biofilm infections. In vitro and in vivo experiments demonstrated that the minimum inhibitory concentration (MIC) and the minimum bactericidal concentration (MBC) for biofilm bacterial cells were usually much higher (approximately 10-1 000 times) than the planktonic bacterial cells. ${ }^{4-6}$ The effective antibiotic $\mathrm{MBC}$ in vivo for biofilm eradication are therefore impossible to reach by conventional antibiotic administrations due to the toxicities and the side effects of antibiotics and the limitation of renal and hepatic functions. Treatment of biofilm infections becomes therefore challenging and attracts significantly scientific attention. Numerous of

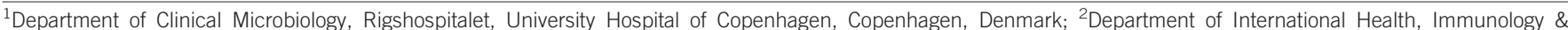
Microbiology, University of Copenhagen, Copenhagen, Denmark and ${ }^{3}$ Department of Clinical Microbiology, Slagelse Hospital, Slagelse, Denmark

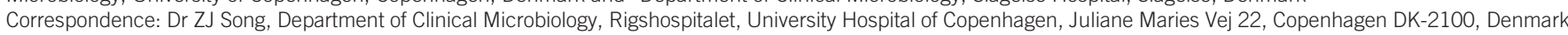
E-mail: szj56@hotmail.com

Accepted 16 July 2014 
clinical investigations have been performed, which would benefit the control of biofilm. The review would focus mainly on the clinical treatment of bacterial biofilm infections based on the achievements in biofilm researches in combination with our clinical experiences.

\section{Diagnosis of biofilm infections}

Clinical evidences of biofilm infection. According to the features of biofilm development, mature biofilms are significantly resistant to antibiotic chemotherapies and they will intermittently disperse planktonic bacterial cells to the environments. Hence, a typical biofilm infection is usually a chronic infection with intermittent exacerbations; antibiotic treatments could be helpful to control the acute exacerbations, but difficult to eradicate the infection. Generally biofilm infection could be suspected, if a patient has one of the clinical manifestations shown in Table 1.

Routine microbiological examinations. Traditional microbiological examination includes sample collection, microbial cultivation, identification and tests of antibiotic susceptibilities, in which appropriate sample collection is essential according to our clinical experiences. For example, in patients suspected for foreign body-associated biofilm infections, at least 4-5 pieces of tissue biopsy from different sites next to the prosthesis suspected infection are needed to avoid a false negative result. The prostheses, catheters or stents and other foreign bodies taken out from patients due to suspicion of biofilm infections should be sent for microbiological examinations. For the microscopy and culture-negative samples, if the patients are highly suspected for biofilm infections clinically, additional microbiological techniques might be helpful for the diagnosis of biofilm infections.

New techniques of microbiology. Routine microbiological examinations are important and reliable for diagnosis of infections, but somehow less sensitive for biofilm detection. Therefore new techniques of microbiology should be introduced as efficient complements of routine microbiology or part of the novel routine methods in hospitals.

It has been proved that proper sonication of indwelling devices (implants or prostheses or catheters) from the patients with suspected infection could significantly improve the detection rate of bacteria. $^{13,28-29}$ In microscopy and culture negative samples from the patients with clinical suspicion of biofilm infection, a 16S ribosomal RNA polymerase chain reaction (PCR) (bacteria) or $18 \mathrm{~S}$ and $28 \mathrm{~S}$ rRNA PCR (fungi) examinations could be appreciated, ${ }^{30-31}$ which have been applied as part of our laboratory examinations for several years. In addition, peptide nucleic acid fluorescence in situ hybridization has been demonstrated useful to identify biofilm infections in cystic fibrosis and chronic wounds. ${ }^{26,32}$ Besides bacteria detection, fluorescence in situ hybridization is highly sensitive in invasive yeast infections, but less sensitive for moulds. ${ }^{33}$

\section{TREATMENT OF MICROBIAL BIOFILM INFECTIONS}

As reported in several papers, that biofilm infections are difficult to handle and are often antibiotic treatment alone inadequate. Generally, the strategies can be divided into involving a foreign body or not. If not involving a foreign body, long-term treatment with high doses and often using combination of antibiotics with different killing mechanisms can sometimes eliminate the infection. However, if a foreign body is involved, removal of the material is in most cases necessary for a successful outcome. In other cases, only reduction of the biofilm is possible followed by chronic biofilm suppressive treatment or waiting for the biofilm to relapse with an exacerbation. Here we would like to share our clinical experiences with our colleagues in combination with the latest relevant literature.

\section{Removal of foreign bodies and abscess}

It has been demonstrated that high inoculums $\left(10^{8} \mathrm{CFU} \cdot \mathrm{mL}^{-1}\right.$; CFU, colony forming units) of Staphylococcus aureus in animal soft tissues could not create any abscesses in the absence of foreign body, whereas $10^{2} \mathrm{CFU} \cdot \mathrm{mL}^{-1}$ of $\mathrm{S}$. aureus were sufficient to induce an infection with foreign body in $95 \%$ of the cases despite significant presence of polymorphonuclear leukocytes, ${ }^{34}$ and this might be associated with the fact that the presence of foreign body significantly downregulated the phagocytosis and intracellular bactericidal effects of polymorphonuclear leukocytes. ${ }^{35}$ Obviously, foreign body provides an ideal surface for bacteria to attach to, whereas polymorphonuclear leukocyte functions are injured due to the presence of foreign body. Thus, the presence of foreign body increased significantly the possibility of biofilm infection. According to the biofilm characters of antibiotic resistance, it is currently difficult to eradicate biofilm infections by conventional antibiotic treatments. Therefore, the removal of a foreign body becomes an important prerequisite for the eradication of such biofilm infections. It is thus highly recommended to remove the infected indwelling devices implanted into patients for medical reasons or replace the infected device with a new one, if we hope to cure the biofilm infections. In case not possible to remove the infected foreign body, an attempt to reduce the biofilm burden with antibiotics followed by continued suppressive antibiotic treatment to prevent regrowth of the biofilm could be suggested.

Table 1 Clinical signs of suspected biofilm infections

\begin{tabular}{|c|c|c|}
\hline Possible biofilm infections & Clinical manifestations and paraclinical changes & The common pathogens \\
\hline Endocarditis & $\begin{array}{l}\text { Patients equipping with or without prosthetic heart valves or pacemaker, who have intermittent } \\
\text { fever and bacteremia with an identical pathogen and without an obvious focus, but higher } \\
\text { C-reaction proteins and/or erythrocyte sedimentation rate with or without leukocytosis }{ }^{88-89}\end{array}$ & $\begin{array}{l}\text { S. aureus, Streptococcus species, coagulase- } \\
\text { negative staphylococci, Enterococcus species }\end{array}$ \\
\hline $\begin{array}{l}P . \text { aeruginosa biofilm in } \\
\text { CF/COPD }\end{array}$ & Patients with CF or COPD, who have been detected mucoid $P$. aeruginosa in sputum ${ }^{4}$ & $P$. aeruginosa \\
\hline Intravenous catheter biofilm & $\begin{array}{l}\text { Patients with central venous catheter or hemodialysis catheter, who have recurrent } \\
\text { bacteraemia with an identical pathogen }{ }^{12,90}\end{array}$ & Coagulase-negative staphylococci \\
\hline Urinary catheter biofilm & $\begin{array}{l}\text { Patients with urinary catheter, who have recurrent urinary tract infections } \\
\text { with the same pathogen }{ }^{12,90}\end{array}$ & $\begin{array}{l}\text { Gram-negative rods, Candida species, } \\
\text { Enterococcus species }\end{array}$ \\
\hline $\begin{array}{l}\text { Biofilm infections of } \\
\text { orthopaedics }\end{array}$ & $\begin{array}{l}\text { Patients with joint prostheses or orthopedic fixation devices, who have chronic pain } \\
\text { locally and sign of prostheses loosening }{ }^{13}\end{array}$ & S. aureus, coagulase-negative staphylococci \\
\hline Wound biofilm & Patients with chronic wound and recurrent wound infections ${ }^{25-26}$ & S. aureus, $P$. aeruginosa \\
\hline
\end{tabular}

CF, cystic fibrosis; COPD, chronic obstructive pulmonary diseases. 
Change of the infected central venous catheter (CVC) or dialysis catheter. When bacteria form biofilm on CVC or dialysis catheter, an intermittent bacteraemia with an identical bacterial stain could be expected. In addition, the positive rate of blood cultures sampled from the infected catheter is usually higher than that from the peripheral veins and the time to positivity is at least two hours shorter if the blood is taken through a CVC containing a biofilm compared to a simultaneously blood culture taken through a peripheral vein. ${ }^{36-37}$ To cure such catheter biofilm infections, change of the infected catheter is crucial, followed by a short time treatment of sensitive antibiotic intravenously to remove the bacteria released into blood stream from the infected catheter. In case change of catheter is not possible temporarily, antibiotic and other lock therapy may help to minimize the release of planktonic bacterial cells from the catheter biofilm, which means instillation of high concentrations of antibiotic with or without anti-coagulant or $70 \%$ ethanol or hydrochloric acid $\left(2 \mathrm{~mol} \cdot \mathrm{L}^{-1} \mathrm{HCl}\right)$ into the lumen of CVC. $^{38-41}$ In our clinical practice, vancomycin $\left(1 \mathrm{mg} \cdot \mathrm{mL}^{-1}\right)$ is used to the catheter infection with Gram-positive bacteria and gentamicin $\left(2 \mathrm{mg} \cdot \mathrm{L}^{-1}\right)$ is used to the Gram-negative bacteria. In alternative, $70 \%$ ethanol or $2 \mathrm{~mol} \cdot \mathrm{L}^{-1} \mathrm{HCl}$ lock therapy can also be considered.

Change of the infected urinary catheter (UC). Catheter-associated urinary tract infections are the most common nosocomial infection, which associated with the formation of microbial biofilm in UC. In addition to intermittent urinary tract infections with the identical pathogen, it can also result in urosepsis. Change of the infected UC is not difficult; however, the time to change is important. It is recommended to change the infected UC after 48 h of adequate and sensitive antibiotic treatment to minimize the bacterial concentration in bladder and urinary tract; otherwise, the new UC would be colonized quickly by the bacteria to form new biofilm.

Change of the infected joint prostheses. Prosthesis-related infection is a serious complication in patients with joint replacement and it has been demonstrated as a biofilm correlated infection with poor prognosis. ${ }^{13,42}$ In case the prosthesis infection is diagnosed, change of the infected prosthesis in most of the cases becomes the only choice. If the prosthetic implants are loosening due to biofilm infection, staged exchange of prosthesis in combination with sensitive and aggressive antibiotic treatment is recommended. ${ }^{13,42}$

Changes of other infected indwelling devices. Endocarditic patients with prosthetic heart valves or cardiac pacemakers are at risk of intermittent sepsis, cardiac insufficiency and infective embolic complications. ${ }^{43-44}$ Therefore, change of the infected prosthetic heart valves or cardiac pacemakers in combination with aggressive and sensitive antibiotic therapy becomes necessary. ${ }^{44}$ For the patients with biofilm infections in biliary stents, endotracheal tubes, dead bones (chronic osteomyelitis), biliary and urinary stones (biliary and urinary tract infections), effective antibiotic treatments and removal of the infected foreign bodies are crucial to cure the infections.

Empty of abscesses. Abscesses are not biofilm, but they have some kinds of connections with biofilm. ${ }^{45}$ When an abscess is formed, it becomes difficult for antibiotic to penetrate through the wall of abscess into the focus. Therefore empty of abscess is necessary.

Early and aggressive antibiotic treatments against biofilm infections In vitro experiment showed that young biofilm could be easily cleared by antibiotic treatment compared to the matured biofilm. ${ }^{5}$ Therefore early and aggressive antibiotic treatments are recommended for biofilm infections. ${ }^{4}$ However, early diagnosis of biofilm infection is currently difficult and most of the clinical biofilm infections are actually matured biofilms which are usually difficult to eradicate with antibiotic treatment. ${ }^{4-6,46}$ It is therefore important and crucial to legitimately apply currently available antibiotics in the treatment of biofilm infections. On the basis of removing foreign bodies and combined with the results from our previous studies, ${ }^{3-6,8,13,46}$ the following factors should be taken into account when an antibiotic treatment against biofilm infection is to be decided:

Selection of antibiotics. Treatment of biofilm infection requires sensitive and well-penetrating antibiotics to ensure a sufficient concentration of effective antibiotic at the site of biofilm infection. In general, macrolides, lincosamides, tetracyclines, rifamycins, quinolones, fusidic acid, nitroimidazole, sulfonamides and oxazolidinones penetrate better in tissues and cells than beta-lactam (including penicillins, cephalosporins and carbapenems), aminoglycosides, glycopeptide and polymyxin. It is well known that infection could lead to inflammation, which results in faster metabolism and significant consumption of oxygen locally or systemically. If oxygen supply could not meet the demand, glycolysis will be activated leading to acidosis, and the effects of antibiotics could be affected by $\mathrm{pH}$ values. It has been reported previously that low $\mathrm{pH}$ value $(\mathrm{pH} 5.2)$ could decrease the effects of $\beta$-lactam antibiotics and increase effects of rifamycin SV. ${ }^{47}$ Therefore antibiotic treatment and correction of acid-base balance disorders could be important for the treatment of biofilm infections.

Administration of antibiotics. We have previously demonstrated that combination therapy of antibiotics against biofilm infection was significantly better than antibiotic monotherapy. ${ }^{48}$ Antibiotic combination therapy is therefore recommended for the treatment of biofilm infections. According to the character of antibiotic tolerance and resistance in biofilm and the high MIC and MBC of biofilm cells demonstrated in experimental studies, ${ }^{5-6}$ high dosages of antibiotics under the safe range of renal and hepatic functions are suggested. In addition, a proper duration of antibiotic treatment is also important. For the patients with biofilm infections suitable for topical treatment of high concentrations of antibiotics, systemic combined with topical antibiotic treatment can give better effects against biofilm infections, such as antibiotic inhalation or direct administration for airway biofilms ${ }^{8,49}$ and bladder irrigation with high concentration of antibiotics against biofilm urinary tract infections.

The pharmacokinetics (PK) and pharmacodynamics (PD) of antibiotics in biofilm infections. Bacteria growing in a biofilm could become 10-1000 times more resistant and tolerant to antibiotics compared with their planktonic counterpart. ${ }^{50-51}$ Antimicrobials available for the treatment of highly resistant bacterial infections are limited; ${ }^{52-53}$ therefore, dosage optimization of currently available antibiotics becomes extremely important to improve anti-infection outcomes and to prevent further development of antimicrobial resistance and tolerance. The PK and PD of antimicrobial agents can be used reliably to predict the effect of antimicrobial regimens to achieve maximum bactericidal effect against infections. Several recent studies have shown the different $\mathrm{PK}$ and $\mathrm{PD}$ profiles of antibiotics between planktonic and biofilm infection..$^{5-6,54} \mathrm{PK}$ and PD information of antimicrobial agents on biofilm-associated bacteria can be applied to optimize the dose regimens on biofilm infections. ${ }^{6}$ 
Minimum biofilm inhibitory concentration (MBIC) and minimum biofilm eradication concentration are two PD parameters for antimicrobials in biofilm infections. ${ }^{5}$ The application of biofilm growing bacteria in the susceptibility tests of clinical laboratory, with MBIC and minimum biofilm eradication concentration, is useful to obtain a better outcome of antimicrobial chemotherapy, compared with the traditional susceptibility test based on planktonic bacteria. ${ }^{5}$ In our previous PK/PD study, colistin showed a concentration-dependent killing, and imipenem showed a time-dependent killing on biofilm bacteria in vivo. ${ }^{6}$ The elimination of $P$. aeruginosa biofilm bacteria in the lungs of our experimentally infected animals was best correlated to AUC/MBIC of colistin (AUC, the area under the concentrationtime curve), and $\mathrm{T}>\mathrm{MBIC}$ of imipenem ( $\mathrm{T}>\mathrm{MBIC}$, the duration of time a drug concentration remains above the MBIC). ${ }^{6}$

\section{SCIENTIFIC PERSPECTIVES FOR THE CONTROL OF BIOFILM INFECTIONS}

Microbes must be able to sense their surviving environments and modify their physiological processes in order to adapt and thereby survive better. It has been demonstrated that quorum sensing (QS) functions as one of the most important global regulations in bacteria and fungi. ${ }^{55}$ Bacteria use QS to coordinate gene expression according to their density, which functions as a decision-making process to regulate the production of virulent factors and create infection. Another popular target has been raised in recent years, i.e., nucleotide signaling, in which nucleotides are considered as second messengers, including cyclic diguanosine monophosphate (c-di-GMP), cyclic diadenosine monophosphate (c-di-AMP), cyclic guanosine monophosphate (cGMP), cyclic adenosine monophosphate (cAMP) and guanosine tetraphosphate (ppGpp)..$^{56}$ Nucleotide signaling controls several key processes required for bacterial adaptation, and may implicate in pathogenicity as QS. Among the above several nucleotides, c-di-GMP has attracted great attention since it is involved closely in biofilm formation in Gram-negative bacteria. ${ }^{57-58}$ Therefore, the control of quorum sensing and the modification of c-di-GMP become the drug objectives for the development of new anti-biofilm drugs. Beside of the focus on QS and c-di-GMP, bacterial amyloids have become another popular topic. Amyloids has been identified in both bacteria and fungi, since many types of bacterial species relay on amyloids to stick to each other or further to host surfaces resulting in the creation of biofilms. Damage of amyloid structures might give a new concept to control bacterial biofilms. ${ }^{59}$

\section{QUORUM SENSING AS TARGET TO CONTROL BIOFILM INFECTION}

\section{QS inhibitors and anti-QS peptides}

It was well demonstrated 10 years ago that target of QS with synthetic furanones significantly attenuated the lung infections of $P$. aeruginosa in vivo. ${ }^{60}$ The recent analyses of synthetic molecules by $\mathrm{O}^{\prime}$ Loughlin et al. ${ }^{61}$ disclosed the inhibition of the two P. aeruginosa QS receptors, LasR and RhlR by synthetics. Their most effective compound, metabromo-thiolactone, significantly inhibits the production of virulence factor pyocyanin and biofilm formation. Caenorhabditis elegans and human lung epithelial cells were protected from the killing of $P$. aeruginosa by treatment with meta-bromo-thiolactone. They further found the relevant target was RhlR, not LasR in vivo. It has been confirmed in guinea pigs study that a novel QS inhibitor coded as 'yd 47', showed an effect against otitis media and biofilm formation induced by $S$. pneumoniae on Cochlear implants. ${ }^{62}$ The combination of QS inhibitor FS3 and daptomycin was investigated for the prevention of prosthesis biofilm in a rat model of staphylococcal vascular graft infection. Both values of MIC and MBC for daptomycin were lower in the presence of FS3 at an in vitro study. The combination of FS3 and daptomycin exhibited significant synergy efficacy when compared to any single treatment. ${ }^{63}$

RNAIII-inhibiting peptide was reported to suppress staphylococcal TRAP/agr systems and to reduce biofilm formation in vivo. The results indicate the importance of quorum sensing in biofilm infection in the host. The treatment with RNAIII-inhibiting peptide in rats has been found to strongly prevent methicillin-resistant $S$. aureus graft infections, and suggesting that RNAIII-inhibiting peptide can be expected as an anti-QS or/and anti-biofilm agent. ${ }^{64}$ LoVetri and Madhyastha ${ }^{65}$ reported the effects of anti-QS peptides and analogs on the growth of biofilm formation in oral bacteria. It is interesting that a natural QS peptide, competence-stimulating peptide, produced by Streptococcus mutans, could kill their own cells at higher concentrations than normal. In addition to cells-killing, KBI-3221, an analog of competencestimulating peptide developed by various Streptococcus species, was shown to decrease biofilm formation.

Attenuation of bacterial QS by furanones, ginseng, garlic and azithromycin significantly improved the immune clearance and the effects of antibiotics in vitro and in the animal models of $P$. aeruginosa biofilm pneumonia. ${ }^{60,66-69}$ Brackman et al. ${ }^{70}$ demonstrated that QS inhibitor increased the susceptibilities of both Gram-positive and -negative bacterial biofilms to antibiotics in vitro and in vivo. Azithromycin has been actually applied routinely to the CF patients as an anti-QS treatment in several CF centers around the world including the Danish CF Center in Copenhagen.

\section{Modification of c-di-GMP as target to disperse biofilm infections} C-di-GMP was discovered 25 years ago, and has been emerged as one of the most common and important bacterial second messengers. Cdi-GMP has been shown to play key roles in lifestyle changes of many bacteria, for example, transforming from the motile to the sessile state to establish multicellular biofilm communities, and change from the virulent state of acute infections to the less virulent but chronic infections. Therefore, modulating c-di-GMP signalling pathways in bacteria could offer a new way to manage the formation and dispersal of biofilms in clinic situations. ${ }^{58}$

The inhibitors of diguanylate cyclase (DGC), the enzyme that synthesizes c-di-GMP were identified by Palys group. They found four small molecules functioning as antagonists of DGC and had shown impairments on the biofilms formed by $P$. aeruginosa and Acinetobacter baumannii. The biofilms of $P$. aeruginosa developed on urinary catheters could be dispersed and inhibited by all four molecules. Two of the four screened molecules displayed no toxic effects on eukaryotic cells, which open a potential imagination to control biofilm infections. ${ }^{71}$

Another discovery based on the differential radial capillary action of ligand assay was able to identify small molecules that inhibit c-di-GMP binding to the allosteric sites. It was found that ebselen reduced DGC activity, and ebselen oxide, the selenone analog of ebselen, also inhibited c-di-GMP binding through the same covalent mechanism. The results confirmed that ebselen and ebselen oxide through inhibition of DGCs limited c-di-GMP in regulation of biofilm formation in $P$. aeruginosa. ${ }^{72}$

Our experimental studies demonstrated that Chinese ginseng could inhibit the QS of P. aeruginosa, and also induce dispersal of $P$. aeruginosa biofilm in vitro by means of activating motilities of the bacterium. ${ }^{68,73}$ Connolly et al. ${ }^{74}$ found that the cysteine protease SpeB could induce dispersal in Group A streptococcal biofilm. Park et al. ${ }^{75}$ 
reported that the proteases from $P$. aeruginosa could inhibit the biofilm formation and result in biofilm dispersal in $S$. aureus.

\section{Disruption of bacterial amyloids to control bacterial biofilms}

Many bacteria can constitute functional amyloid fibers on their cell surface. The majority of bacterial amyloids contribute to the development of biofilm as well as other community behaviors. Curli are functional extracellular amyloid fibers produced by Escherichia coli and other Enterobacteriaceae. Two analogs of FN075 and BibC6 of ringfused 2-pyridones, the peptidomimetics that target essential protein-protein interactions in macromolecular assembly, inhibited curli biogenesis in E. coli and further pre-treatment of E. coli with FN075 significantly attenuated virulence in a mouse model of urinary tract infection. Curli and type 1 pili exhibited exclusive and independent roles in promoting E. coli biofilms; thus, the ability of FN075 to block the biogenesis of both curli and type 1 pili composes unique antibiofilm and anti-virulence activities on these compounds. ${ }^{76}$

It has been shown recently that Bacillus subtilis biofilms can be inhibited by controlling the formation of amyloid-like fibers with the two molecules of AA-861 (a benzoquinone derivative) and parthenolide (a sesquiterpene lactone), which were screened from among a collection of hundreds of known bioactive molecules. The findings concluded that AA-861 prevented the TasA protein from forming functional amyloid-like fibers. Parthenolide as a natural product showed also an effect of disrupting pre-established biofilms. In addition, the molecules prevent the formation of biofilms of other bacterial species that could secrete amyloid proteins. ${ }^{77}$

Sintim et al. ${ }^{78}$ summarized a group of small molecules that could potentially attenuate bacterial virulence or inhibit biofilm formation. However, there are no US FDA-approved molecules that have been discovered to target these processes. Therefore, we will face with a 'chronic and severe' course just as the infections caused by bacterial biofilms.

\section{Bacteriophage therapies}

Bacteriophages are viruses that infect and replicate within bacteria and either become lysogenic or lyse (kill) the host bacteria. Bacteriophage therapy for bacterial infections has been used for more than 50 years and the development of antibiotic resistance, especially the multidrugresistant bacteria attracts more attention to the investigation of bacteriophages. ${ }^{79}$ Experimental and clinical studies have shown exciting effects in not only wound biofilm infections, but also implant- and catheter-related infections. ${ }^{79-84}$ Bacteriophages are able to infect and kill both of the antibiotic-sensitive and -resistant bacteria.

\section{Other new findings}

Singh et al. ${ }^{85}$ reported that low concentration of lactoferrin, a component of innate immunity blocked the development of $P$. aeruginosa biofilm by stimulating the twitching movement of the bacterium. Similarly, aqueous extract of Chinese ginseng hindered the formation of $P$. aeruginosa biofilm by means of enhancing the swimming and twitching motility and reducing the swarming motility. ${ }^{73}$ Japanese researchers found that Esp, a serine protease secreted by Staphylococcus epidermidis could inhibit the formation of $S$. aureus biofilm and destroy the preformed $S$. aureus biofilm in vitro and inhibit the nasal colonization of $S$. aureus in vivo, ${ }^{86}$ indicating the potential effects of commensal bacteria. It has been demonstrated that ultrasoundtargeted microbubble destruction could significantly improve vancomycin against $S$. epidermidis RP62A biofilms. ${ }^{87}$ The biofilms treated with vancomycin plus ultrasound-targeted microbubble destruction showed significantly decreased biofilm densities and the viable counts of S. epidermidis compared with those groups treated with vancomycin or ultrasound-targeted microbubble destruction alone. ${ }^{87}$ The combination with alternative therapies may have great potential to enhance antibiotic treatment in bacterial biofilm infections.

\section{CONCLUSION}

Treatment of biofilm infections is currently a difficult and complicated challenge for microbiologists and clinicians. Antibiotic treatment alone is often inadequate to overcome biofilm infections. However, the progresses of research provide us with more detailed inside knowledge to better understand the nature of microbial biofilms, which has benefited and will continue to support our efforts of combating biofilm infections. Currently, treatment of biofilm infections needs collaboration in clinical microbiology, surgery, internal medicine, pharmacology and basic science, i.e., a multidisciplinary cooperation. We believe that biofilm treatment at present should include removal of infected indwelling devices, selection of well penetrating and sensitive antibiotics, early administration of high dosage antibiotics in combination and supplemented with anti-QS treatment and/or biofilm dispersal agents.

\section{ACKNOWLEDGEMENTS}

Publication of this manuscript is supported by Open Fund of State Key Laboratory of Oral Diseases, Sichuan University.

1 de Fuente-Núñez C, Reffuveille F, Fernandez L et al. Bacterial biofilm development as a multicellular adaptation: antibiotic resistance and new therapeutic strategies. Curr Opin Microbiol 2013; 16(5): 580-589.

2 Hall-Stoodley L, Costerton JW, Stoodley P. Bacterial biofilms: from the natural environment to infectious diseases. Nat Rev Microbio/ 2004; 2(2): 95-108.

3 Yang L, Liu Y, Wu H et al. Combating biofilms. FEMS Immunol Med Microbiol 2012; 65(2): 146-157

4 Høiby N, Ciofu O, Johansen HK et al. The clinical impact of bacterial biofilms. Int J Oral Sci 2011; 3(2): 55-65.

5 Hengzhuang W, Wu H, Ciofu $\mathrm{O}$ et al. Pharmacokinetics/pharmacodynamics of colistin and imipenem on mucoid and nonmucoid Pseudomonas aeruginosa biofilms. Antimicrob Agents Chemother 2011; 55(9): 4469-4474.

6 Hengzhuang W, Wu H, Ciofu $\mathrm{O}$ et al. In vivo pharmacokinetics/pharmacodynamics of colistin and imipenem in Pseudomonas aeruginosa biofilm infection. Antimicrob Agents Chemother 2012; 56(5): 2683-2690.

7 Høiby N, Bjarnsholt T, Givskov M et al. Antibiotic resistance of bacterial biofilms. Int J Antimicrob Agents 2010; 35(4): 322-332.

$8 \mathrm{H} \varnothing i b y \mathrm{~N}$. Recent advances in the treatment of Pseudomonas aeruginosa infections in cystic fibrosis. BMC Med 2011; 9: 32.

9 Tran PL, Lowry N, Campbell T et al. An organoselenium compound inhibits Staphylococcus aureus biofilms on hemodialysis catheters in vivo. Antimicrob Agents Chemother 2012; 56(2): 972-978.

10 Tollefson DF, Bandyk DF, Kaebnick HW et al. Surface biofilm disruption. Enhanced recovery of microorganisms from vascular prostheses. Arch Surg 1987; 122(1): 3843.

11 Fux CA, Quigley M, Worel AM et al. Biofilm-related infections of cerebrospinal fluid shunts. Clin Microbiol Infect 2006; 12(4): 331-337.

12 Donlan RM. Biofilms and device-associated infections. Emerg Infect Dis 2001; 7(2): 277-281.

13 Song Z, Borgwardt L, Høiby $\mathrm{N}$ et al. Prosthesis infections after orthopedic joint replacement: the possible role of bacterial biofilms. Orthop Rev (Pavia) 2013; 5(2): 65-71.

14 Santos AP, Watanabe E, Andrade D. Biofilm on artificial pacemaker: fiction or reality? Arq Bras Cardiol 2011; 97(5): e113-e120.

15 Dasgupta MK. Biofilms and infection in dialysis patients. Semin Dial 2002; 15(5): 338-346.

16 Auler ME, Morreira D, Rodrigues FF et al. Biofilm formation on intrauterine devices in patients with recurrent vulvovaginal candidiasis. Med Mycol 2010; 48(1): 211-216.

17 Donelli G, Vuotto C, Cardines R et al. Biofilm-growing intestinal anaerobic bacteria. FEMS Immunol Med Microbiol 2012; 65(2): 318-325.

18 Murakami M, Nishi Y, Seto K et al. Dry mouth and denture plaque microflora in complete denture and palatal obturator prosthesis wearers. Gerodontology 2013; doi: 10.1111/ger.12073.

19 Rieger UM, Mesina J, Kalbermatten DF et al. Bacterial biofilms and capsular contracture in patients with breast implants. Br J Surg 2013; 100(6): 768-774 
20 Abidi SH, Sherwani SK, Siddiqui TR et al. Drug resistance profile and biofilm forming potential of Pseudomonas aeruginosa isolated from contact lenses in KarachiPakistan. BMC Ophthalmol 2013; 13: 57.

21 Høiby N, Ciofu O, Bjarnsholt T. Pseudomonas aeruginosa biofilms in cystic fibrosis. Future Microbiol 2010; 5(11): 1663-1674.

22 Martinez-Solano L, Macia MD, Fajardo A et al. Chronic Pseudomonas aeruginosa infection in chronic obstructive pulmonary disease. Clin Infect Dis 2008; 47(12): $1526-1533$

23 Wessman M, Bjarnsholt T, Eickhardt-Sorensen SR et al. Mucosal biofilm detection in chronic otitis media: a study of middle ear biopsies from Greenlandic patients. Eur Arch Otorhinolaryngol 2014; doi: 10.1007/s00405-014-2886-9.

24 Jain R, Douglas R. When and how should we treat biofilms in chronic sinusitis? Curr Opin Otolaryngol Head Neck Surg 2014; 22(1): 16-21.

25 Percival SL, Hill KE, Williams DW et al. A review of the scientific evidence for biofilms in wounds. Wound Repair Regen 2012; 20(5): 647-657.

26 Malic S, Hill KE, Hayes A et al. Detection and identification of specific bacteria in wound biofilms using peptide nucleic acid fluorescent in situ hybridization (PNA FISH). Microbiology 2009; 155(Pt 8): 2603-2611.

27 Paredes J, Onso-Arce M, Schmidt C et al. Smart central venous port for early detection of bacterial biofilm related infections. Biomed Microdevices 2014; 16(3): 365-374.

28 Jost GF, Wasner M, Taub E et al. Sonication of catheter tips for improved detection of microorganisms on external ventricular drains and ventriculo-peritoneal shunts. J Clin Neurosci 2013; 21(4): 578-582.

29 Portillo ME, Salvado M, Trampuz A et al. Sonication versus vortexing of implants for diagnosis of prosthetic joint infection. J Clin Microbiol 2013; 51(2): 591-594.

30 Guembe M, Marin M, Martin-Rabadan P et al. Use of universal 16S rRNA gene PCR as a diagnostic tool for venous access port-related bloodstream infections. J Clin Microbiol 2013; 51(3): 799-804.

31 Khot PD, Ko DL, Fredricks DN. Sequencing and analysis of fungal rRNA operons for development of broad-range fungal PCR assays. Appl Environ Microbiol 2009; 75(6): 1559-1565.

32 Bjarnsholt T, Nielsen XC, Johansen $\mathrm{U}$ et al. Methods to classify bacterial pathogens in cystic fibrosis. Methods Mol Biol 2011; 742: 143-171.

33 Rickerts V, Khot PD, Myerson D et al. Comparison of quantitative real time PCR with Sequencing and ribosomal RNA-FISH for the identification of fungi in formalin fixed, paraffin-embedded tissue specimens. BMC Infect Dis 2011; 11: 202.

34 Zimmerli W, Waldvogel FA, Vaudaux $\mathrm{P}$ et al. Pathogenesis of foreign body infection: description and characteristics of an animal model. J Infect Dis 1982; 146(4): 487497.

35 Zimmerli W, Lew PD, Waldvogel FA. Pathogenesis of foreign body infection. Evidence for a local granulocyte defect. J Clin Invest 1984: 73(4): 1191-1200.

36 Raad II, Hanna HA. Intravascular catheter-related infections: new horizons and recent advances. Arch Intern Med 2002; 162(8): 871-878.

37 Mermel LA, Allon M, Bouza E et al. Clinical practice guidelines for the diagnosis and management of intravascular catheter-related infection: 2009 Update by the Infectious Diseases Society of America. Clin Infect Dis 2009; 49(1): 1-45.

38 Fernandez-Hidalgo N, Almirante B. Antibiotic-lock therapy: a clinical viewpoint. Expert Rev Anti Infect Ther 2014; 12(1): 117-129.

39 Vandenhende MA, Buret J, Camou F et al. Successful daptomycin lock therapy for implantable intra-arterial catheter infection in a patient with liver metastases of colon cancer. Diagn Microbiol Infect Dis 2014; 78(4): 497-498.

40 Tan M, Lau J, Guglielmo BJ. Ethanol locks in the prevention and treatment of catheterrelated bloodstream infections. Ann Pharmacother 2014; 48(5): 607-615.

41 Madsen M, Rosthoj S. Impact of hydrochloric acid instillation on salvage of infected central venous catheters in children with acute lymphoblastic leukaemia. Scand J Infect Dis 2013; 45(1): 38-44.

42 Zimmerli W, Moser C. Pathogenesis and treatment concepts of orthopaedic biofilm infections. FEMS Immunol Med Microbiol 2012; 65(2): 158-168.

43 Mocchegiani R, Nataloni M. Complications of infective endocarditis. Cardiovasc Hematol Disord Drug Targets 2009; 9(4): 240-248.

44 Nataloni M, Pergolini M, Rescigno G et al. Prosthetic valve endocarditis. J Cardiovasc Med (Hagerstown) 2010; 11(12): 869-883.

45 May JG, Shah P, Sachdeva L et al. Potential role of biofilms in deep cervical abscess. Int J Pediatr Otorhinolaryngol 2014; 78(1): 10-13.

$46 \mathrm{H}$ øiby N, Krogh JH, Moser $\mathrm{C}$ et al. Pseudomonas aeruginosa and the in vitro and in vivo biofilm mode of growth. Microbes Infect 2001; 3(1): 23-35.

47 Laub R, Schneider YJ, Trouet A. Antibiotic susceptibility of Salmonella spp. at different pH values. J Gen Microbiol 1989; 135(6): 1407-1416.

48 Herrmann G, Yang L, Wu H et al. Colistin-tobramycin combinations are superior to monotherapy concerning the killing of biofilm Pseudomonas aeruginosa. J Infect Dis 2010; 202(10): 1585-1592.

49 Song Z, Wu H, Mygind P et al. Effects of intratracheal administration of novispirin G10 on a rat model of mucoid Pseudomonas aeruginosa lung infection. Antimicrob Agents Chemother 2005; 49(9): 3868-3874.

50 Ceri H, Olson ME, Stremick C et al. The calgary biofilm device: new technology for rapid determination of antibiotic susceptibilities of bacterial biofilms. JClin Microbiol 1999; 37(6): 1771-1776.

51 Moskowitz SM, Foster JM, Emerson J et al. Clinically feasible biofilm susceptibility assay for isolates of Pseudomonas aeruginosa from patients with cystic fibrosis. J Clin Microbiol 2004; 42(5): 1915-1922.

52 DeRyke CA, Lee SY, Kuti JL et al. Optimising dosing strategies of antibacterials utilising pharmacodynamic principles: impact on the development of resistance. Drugs 2006; 66(1): 1-14.
53 Neu HC. The crisis in antibiotic resistance. Science 1992; 257(5073): 1064-1073.

54 Hengzhuang $W$, Ciofu $O$, Yang $L$ et al. High beta-lactamase levels change the pharmacodynamics of beta-lactam antibiotics in Pseudomonas aeruginosa biofilms. Antimicrob Agents Chemother 2013; 57(1): 196-204.

55 Dubern JF, Diggle SP. Quorum sensing by 2-alkyl-4-quinolones in Pseudomonas aeruginosa and other bacterial species. Mol Biosyst 2008; 4(9): 882-888.

56 Kalia D, Merey G, Nakayama S et al. Nucleotide, c-di-GMP, c-di-AMP, cGMP, cAMP, (p)ppGpp signaling in bacteria and implications in pathogenesis. Chem Soc Rev 2013; 42(1): 305-341.

57 Hengge R. Principles of c-di-GMP signalling in bacteria. Nat Rev Microbiol 2009; 7(4): 263-273.

58 Romling U, Galperin MY, Gomelsky M. Cyclic di-GMP: the first 25 years of a universal bacterial second messenger. Microbiol Mol Biol Rev 2013; 77(1): 1-52.

59 Romero D, Aguilar C, Losick R et al. Amyloid fibers provide structural integrity to Bacillus subtilis biofilms. Proc Natl Acad Sci U S A 2010; 107(5): 22302234.

60 Wu H, Song Z, Hentzer M et al. Synthetic furanones inhibit quorum-sensing and enhance bacterial clearance in Pseudomonas aeruginosa lung infection in mice. $J$ Antimicrob Chemother 2004; 53(6): 1054-1061.

61 O'Loughlin CT, Miller LC, Siryaporn A et al. A quorum-sensing inhibitor blocks Pseudomonas aeruginosa virulence and biofilm formation. Proc Natl Acad Sci U S A 2013; 110(44): 17981-17986

62 Cevizci R, Duzlu M, Dundar $Y$ et al. Preliminary results of a novel quorum sensing inhibitor against pneumococcal infection and biofilm formation with special interest to otitis media and cochlear implantation. Eur Arch Otorhinolaryngol 2014. doi: 10.1007/s00405-014-2942-5. [Epub ahead of print].

63 Cirioni O, Mocchegiani F, Cacciatore I et al. Quorum sensing inhibitor FS3-coated vascular graft enhances daptomycin efficacy in a rat model of staphylococcal infection 2. Peptides $2013 ; 40: 77-81$

64 Balaban N, Cirioni O, Giacometti A et al. Treatment of Staphylococcus aureus biofilm infection by the quorum-sensing inhibitor RIP. Antimicrob Agents Chemother 2007; 51(6): 2226-2229.

65 LoVetri K, Madhyastha S. Antimicrobial and antibiofilm activity of quorum sensing peptides and Peptide analogues against oral biofilm bacteria. Methods Mol Biol 2010; 618: 383-392.

66 Bjarnsholt T, Jensen PO, Rasmussen TB et al. Garlic blocks quorum sensing and promotes rapid clearing of pulmonary Pseudomonas aeruginosa infections. Microbiology 2005; 151(Pt 12): 3873-3880.

67 Hoffmann N, Lee B, Hentzer M et al. Azithromycin blocks quorum sensing and alginate polymer formation and increases the sensitivity to serum and stationary-growth-phase killing of Pseudomonas aeruginosa and attenuates chronic $P$. aeruginosa lung infection in $\mathrm{Cftr}^{-1-}$ mice. Antimicrob Agents Chemother 2007; 51(10): 36773687.

68 Song $\mathrm{Z}$, Kong KF, Wu $\mathrm{H}$ et al. Panax ginseng has anti-infective activity against opportunistic pathogen Pseudomonas aeruginosa by inhibiting quorum sensing, a bacterial communication process critical for establishing infection. Phytomedicine 2010; 17(13): 1040-1046.

$69 \mathrm{Wu} \mathrm{H}$, Song Z, Givskov M et al. Pseudomonas aeruginosa mutations in las/ and rhll quorum sensing systems result in milder chronic lung infection. Microbiology 2001; 147(Pt 5): 1105-1113.

70 Brackman G, Cos P, Maes L et al. Quorum sensing inhibitors increase the susceptibility of bacterial biofilms to antibiotics in vitro and in vivo. Antimicrob Agents Chemother 2011; 55(6): 2655-2661.

71 Sambanthamoorthy K, Luo C, Pattabiraman N et al. Identification of small molecules inhibiting diguanylate cyclases to control bacterial biofilm development. Biofouling 2014; 30(1): 17-28.

72 Lieberman OJ, Orr MW, Wang Y et al. High-throughput screening using the differential radial capillary action of ligand assay identifies ebselen as an inhibitor of diguanylate cyclases. ACS Chem Biol 2014; 9(1): 183-192.

$73 \mathrm{Wu} \mathrm{H}$, Lee B, Yang L et al. Effects of ginseng on Pseudomonas aeruginosa motility and biofilm formation. FEMS Immunol Med Microbiol 2011; 62(1): 49-56.

74 Connolly KL, Roberts AL, Holder RC et al. Dispersal of Group A streptococcal biofilms by the cysteine protease SpeB leads to increased disease severity in a murine model. PLoS One 2011; 6(4): e18984.

75 Park JH, Lee JH, Cho MH et al. Acceleration of protease effect on Staphylococcus aureus biofilm dispersal. FEMS Microbiol Lett 2012; 335(1): 31-38.

76 Cegelski L, Pinkner JS, Hammer ND et al. Small-molecule inhibitors target Escherichia coli amyloid biogenesis and biofilm formation. Nat Chem Biol 2009; 5(12): 913-919.

77 Romero D, Sanabria-Valentin E, Vlamakis $\mathrm{H}$ et al. Biofilm inhibitors that target amyloid proteins. Chem Biol 2013; 20(1): 102-110.

78 Sintim HO, Smith JA, Wang J et al. Paradigm shift in discovering next-generation antiinfective agents: targeting quorum sensing, c-di-GMP signaling and biofilm formation in bacteria with small molecules. Future Med Chem 2010; 2(6): 1005-1035.

79 Soothill J. Use of bacteriophages in the treatment of Pseudomonas aeruginosa infections. Expert Rev Anti Infect Ther 2013; 11(9): 909-915.

80 Burrowes B, Harper DR, Anderson J et al. Bacteriophage therapy: potential uses in the control of antibiotic-resistant pathogens. Expert Rev Anti Infect Ther 2011; 9(9): 775-785.

81 Seth AK, Geringer MR, Nguyen KT et al. Bacteriophage therapy for Staphylococcus aureus biofilm-infected wounds: a new approach to chronic wound care. Plast Reconstr Surg 2013; 131(2): 225-234. 
82 Yilmaz C, Colak M, Yilmaz BC et al. Bacteriophage therapy in implant-related infections: an experimental study. J Bone Joint Surg Am 2013; 95(2): 117-125.

83 Alemayehu D, Casey PG, McAuliffe 0 et al. Bacteriophages phiMR299-2 and phiNH-4 can eliminate Pseudomonas aeruginosa in the murine lung and on cystic fibrosis lung airway cells. MBio 2012; 3(2): e00029-12.

84 Brussow $\mathrm{H}$. Bacteriophage-host interaction: from splendid isolation into a messy reality. Curr Opin Microbiol 2013; 16(4): 500-506.

85 Singh PK, Parsek MR, Greenberg EP et al. A component of innate immunity prevents bacterial biofilm development. Nature 2002; 417(6888): 552-555.

86 Iwase T, Uehara Y, Shinji H et al. Staphylococcus epidermidis Esp inhibits Staphylococcus aureus biofilm formation and nasal colonization. Nature 2010 ; 465(7296): 346-349.

$87 \mathrm{He} \mathrm{N}, \mathrm{Hu}$ J, Liu $\mathrm{H}$ et al. Enhancement of vancomycin activity against biofilms by using ultrasound-targeted microbubble destruction. Antimicrob Agents Chemother 2011; 55(11): 5331-5337.

88 Hoen B, Duval X. Clinical practice. Infective endocarditis. N Engl J Med 2013; 368(15): 1425-1433.
89 Baddour LM, Wilson WR, Bayer AS et al. Infective endocarditis: diagnosis, antimicrobial therapy, and management of complications: a statement for healthcare professionals from the Committee on Rheumatic Fever, Endocarditis, and Kawasaki Disease, Council on Cardiovascular Disease in the Young, and the Councils on Clinical Cardiology, Stroke, and Cardiovascular Surgery and Anesthesia, American Heart Association: endorsed by the Infectious Diseases Society of America. Circulation 2005; 111(23): e394-e434.

90 Smith RN, Nolan JP. Central venous catheters. BMJ 2013; 347: f6570.

(c) (i) $\Theta$ This work is licensed under a Creative Commons Attribution-

c. Non Commercial-NoDerivs 3.0 Unported License. The images or other third party material in this article are included in the article's Creative Commons license, unless indicated otherwise in the credit line; if the material is not included under the Creative Commons license, users will need to obtain permission from the license holder to reproduce the material. To view a copy of this license, visit http://creativecommons.org/licenses/ by-nc-nd/3.0/ 